\title{
Prompts instrucionais como estratégia metacognitiva no ensino de arritmias cardíacas supraventriculares para estudantes de medicina
}

\author{
Instructional prompts as metacognitive strategy for teaching supraventricular arrhyth mias \\ to undergraduate medical students
}

Prompts instruccionales como estrategia metacognitiva para la enseñanza de las arritmias supraventriculares a los estudiantes de medicina

Ricardo Negri Bandeira de Mello ${ }^{1 *}$, Giovanna de Oliveira Fonseca ${ }^{1}$, Amanda Perpetuo de Oliveira ${ }^{1}$, José Maria Peixoto'.

\section{RESUMO}

Objetivo: Comparar o ef eito de dois métodos de ensino (prompts instrucionais e estudo autodirigido) na competência diagnóstica de arritmias supraventriculares em estudantes de medicina. Métodos: Estudo experimental com três fases: avaliação inicial (fase 1), treinamento (fase 2) e avaliação tardia (fase 3). A amostra foi composta por 24 estudantes, que na fase 1 forneceram os diagnósticos dos ritmos cardíacos de 11 traçados de Eletrocardiograma (ECG). Na fase 2, a amostra foi dividida em dois grupos. O Grupo 1 adotou o estudo autodingido, e o Grupo 2 prompts instrucionais. Na fase 3 (após uma semana), toda a amostra forneceu os diagnósticos dos ritmos cardíacos de 11 novos traçados de ECG. Resultados: Após a fase 2, os alunos melhoraram os escores diagnósticos em relação à fase 1 , independentemente o método de ensino utilizado. Na fase 3 , a média das notas do Grupo 2 superou o Grupo $1(\mathrm{p}=0,034)$. Conclusão: $O$ uso de prompts instrucionais no ensino de ritmos cardíacos, foi capaz de melhorar a acurácia diagnóstica dos estudantes para as arritmias estudadas.

Palavras-chave: Educação médica, Método de ensino, Eletrocardiograma, Arritmias cardíacas.

\begin{abstract}
Objective: To compare the effect of two teaching methods (instructional prompts and self -directed study) on diagnostic competence of supraventricular arrhythmias among medical students. Methods: Experimental study with three phases: initial assessment (phase 1), training (phase 2) and late assessment (phase 3). The sample consisted of 24 students, who in phase 1 provided the diagnoses of cardiac rates from 11 Electrocardiogram (ECG) tracings. In phase 2, the sample was divided into two groups. Group 1 adopted the self-directed study, and Group 2 instructional prompts. In phase 3 (one week after), the entire sample provided the diagnoses of cardiac rate in 11 new ECG tracings. Results: After phase 2, students improved diagnostic scores compared to phase 1, regardless of the teaching method used. In phase 3, the average score of Group 2 outperformed Group $1(p=0.034)$. Conclusion: The use of instructional prompts in teaching cardiac rhythms, was able to improve the diagnostic accuracy of students for the studied arrhythmias.
\end{abstract}

Key words: Medical education, Teaching method, Electrocardiogram, Cardiac arrhythmias.

\section{RESUMEN}

Objetivo: Comparar el efecto de dos métodos de enseñanza (prompts instruccionales y estudio autodirigido) sobre la competencia diagnóstica de las arritmias supraventriculares en estudiantes de medicina. Métodos: Estudio experimental con tres fases: evaluación inicial (fase 1), entrenamiento (fase 2) y evaluación tardía (fase 3). La muestra estaba formada por 24 estudiantes, que en la fase 1 proporcionaron los diagnósticos de los ritmos cardíacos de 11 trazados de Electrocardiograma (ECG). En la fase 2, la muestra se dividió en dos grupos. El Gupo 1 adoptó el estudio autodirigido, y el Grupo 2 las indicaciones de instrucción. En la fase 3 (una semana después), toda la muestra proporcionó los diagnósticos de ritmo cardíaco de 11 nuevos trazados de ECG. Resultados: Después de la fase 2, los estudiantes mejoraron las puntuaciones de diagnóstico en comparación con la fase 1 , independientemente del método de enseñanza utilizado. En la fase 3, las puntuaciones medias del Grupo 2 superaron a las del Grupo $1(p=0,034)$. Conclusión: El uso de los prompts instruccionales en la enseñanza de los ritmos cardíacos mejoró la precisión diagnóstica de los estudiantes para las arritmias estudiadas.

Palabras clave: Educación médica, Método de enseñanza, Electrocardiograma, Arritmias cardíacas.

${ }^{1}$ Universidade José do Rosário Vellano (UNIFENAS), Belo Horizonte - MG. *E-mail: ricardonbm@cardiol.br

SUBMETIDO EM: 11/2021

ACEITO EM: 12/2021

PUBLICADO EM: 1/2022

REAS | Vol. 15 (1) | DOI: https://doi.org/10.25248/REAS.e9244.2022 


\section{INTRODUÇÃO}

Até o ano de 2017, as doenças cardiovasculares eram a principal causa de morte no Brasil, respondendo por 30\% de todas as mortes por doenças crônicas não transmissíveis, dado que se aproximava da média global, registrada em 45\% (OLIVEIRA GMM, et al., 2020; NASCIMENTO BR, et al., 2016). Ao registrar a atividade elétrica do coração, o exame de Eletrocardiograma (ECG) permite o diagnóstico rápido de doenças potencialmente fatais, como a isquemia miocárdica e arritmias cardíacas, responsáveis por grande parte das admissões hospitalares, condições que exigem rapidez na interpretação do ECG e boa acurácia diagnóstica (RAUPACH T, et al., 2016; ROJAS FL, et al., 2018; BREEN CJ, et al., 2019).

O ECG é um exame de simples execução, baixo custo e grande utilidade para uma assistência médica segura, especialmente nas urgências e emergências cardiovasculares (BARROS MNDS, et al., 2015). No entanto sua ef etividade como método de suporte às decisões clínicas, depende da capacidade do médico em interpretar corretamente o seu traçado (KRASNE S, et al.,2020).

Estudos têm mostrado que o nível de competência para interpretação do ECG é baixo entre médicos residentes (COOK DA, et al., 2020; ANTIPEROVITCH P, et al., 2018). Há evidências de que nos anos iniciais de suas carreiras, médicos apresentem taxas de erro mais altas que o esperado, levando-se em consideração o nível de responsabilidades que assumem neste momento, quanto passam a atuar em unidades de emergências, de internações e na atenção primária (KRASNE S, et al., 2020). Foi relatado que até 33\% das interpretações do ECG na prática clínica apresentam erros significativos e os estudos que avaliaram a acurácia diagnóstica do ECG, demonstram variações de 25\% a 96\% (BREEN CJ, et al., 2019).

Apesar de ser um dos exames mais utilizados na prática assistencial rotineira, não existe ainda uma metodologia padronizada para o ensino do ECG. Grande variabilidade tem sido relatada tanto em relação à capacitação docente para o ensino, como dos métodos instrucionais utilizados (BREEN CJ, et al., 2019). A interpretação básica do ECG é ensinada como parte do currículo médico da graduação e, em geral, as aulas expositivas tradicionais e as rodas de conversa são os métodos predominantes de instrução (BOJSEN SR, et al., 2015; PAUL B e BARANCHUK A, 2011; BREEN CJ, et al., 2019)

Nota-se na literatura, a relevância dispensada sobre tema do ensino do ECG, que discute as deficiências qualitativas e quantitativas para sua aprendizagem em estudantes de medicina e médicos residentes, além de inúmeras técnicas de ensino que vem sendo testadas com esse objetivo (KOPEC G, et al., 2015, KOPEC G, et al., 2018; BREEN CJ, et al., 2019).

Como para o raciocínio clínico, a interpretação do ECG depende do reconhecimento de padrões, que é um tipo de aprendizado perceptivo. A aprendizagem perceptiva trata da melhoria dos processos cognitivos envolvidos na seleção e extração das informações relevantes em uma tarefa. Este tipo de aprendizagem requer mudanças na forma como a informação é selecionada, atua em conjunto co $m$ os demais processos cognitivos envolvidos na aprendizagem, mas é difícil de ser atingida através de métodos instrucionais convencionais (KRASNE S, et al., 2020).

Para o ensino do raciocínio clínico, diferentes metodologias estão sendo investigadas como: a auto explicação, a reflexão estruturada, o aprendizado por exemplos e o uso de prompts instrucionais $(\mathrm{XU} \mathrm{H}$, et al., 2021; ABREU GCF, et al., 2021). Os prompts são instrumentos didáticos que orientam o estudante a desenvolver uma atividade mediante uma lista de comandos que devem ser cumpridos, podendo ter várias formas e objetivos. Através de perguntas ou dicas indicam ao aluno quando e como se engajar para a solução de um problema, melhorando seus processos de autorregulação e percepção da aprendizagem (MÜLLER NM e SEUFERT T, 2018)

Estudos em raciocínio clínico tem demonstrado as vantagens do uso de prompts na autonomia da aprendizagem, no entanto o uso desta estratégia para o ensino do ECG, tem sido pouco avaliada (CHAMBERLAND M e MAMEDE S, 2015). Tendo em vista a maior influência dos métodos computacionais e híbridos, o estudo dos prompts pode corroborar com o desenvolvimento de estratégias eficientes para o ensino do ECG (VILJOEN CA, et al., 2019; VILJOEN CA, et al., 2020, BARAL R, et al., 2019, KEWCHAROEN J, et al., 2020). 
O fato da habilidade de interpretar o ECG se tratar de uma competência relacionada à expertise diagnóstica, motivou a investigação da aplicabilidade do uso dos prompts instrucionais como método auxiliar para o seu ensino. Para essa finalidade, planejou-se um estudo, que teve como objetivo avaliar o ef eito de duas estratégias de ensino, prompts instrucionais e o estudo autodirigido, na competência diagnóstica de arritmias cardíacas supraventriculares em estudantes de medicina.

\section{MÉTODOS}

\section{Desenho do estudo}

Estudo experimental aplicado no segundo semestre do ano de 2019 com três fases: avaliação inicial (fase 1), treinamento (fase 2) e avaliação tardia (fase 3), que comparou o ef eito do ensino da interpretação eletrocardiográfica de arritmias supraventriculares na competência diagnóstica em estudantes de medicina, entre duas estratégias instrucionais diferentes: uso de prompts instrucionais e o estudo autodirigido (Figura 1).

Figura 1 - Desenho do Estudo.

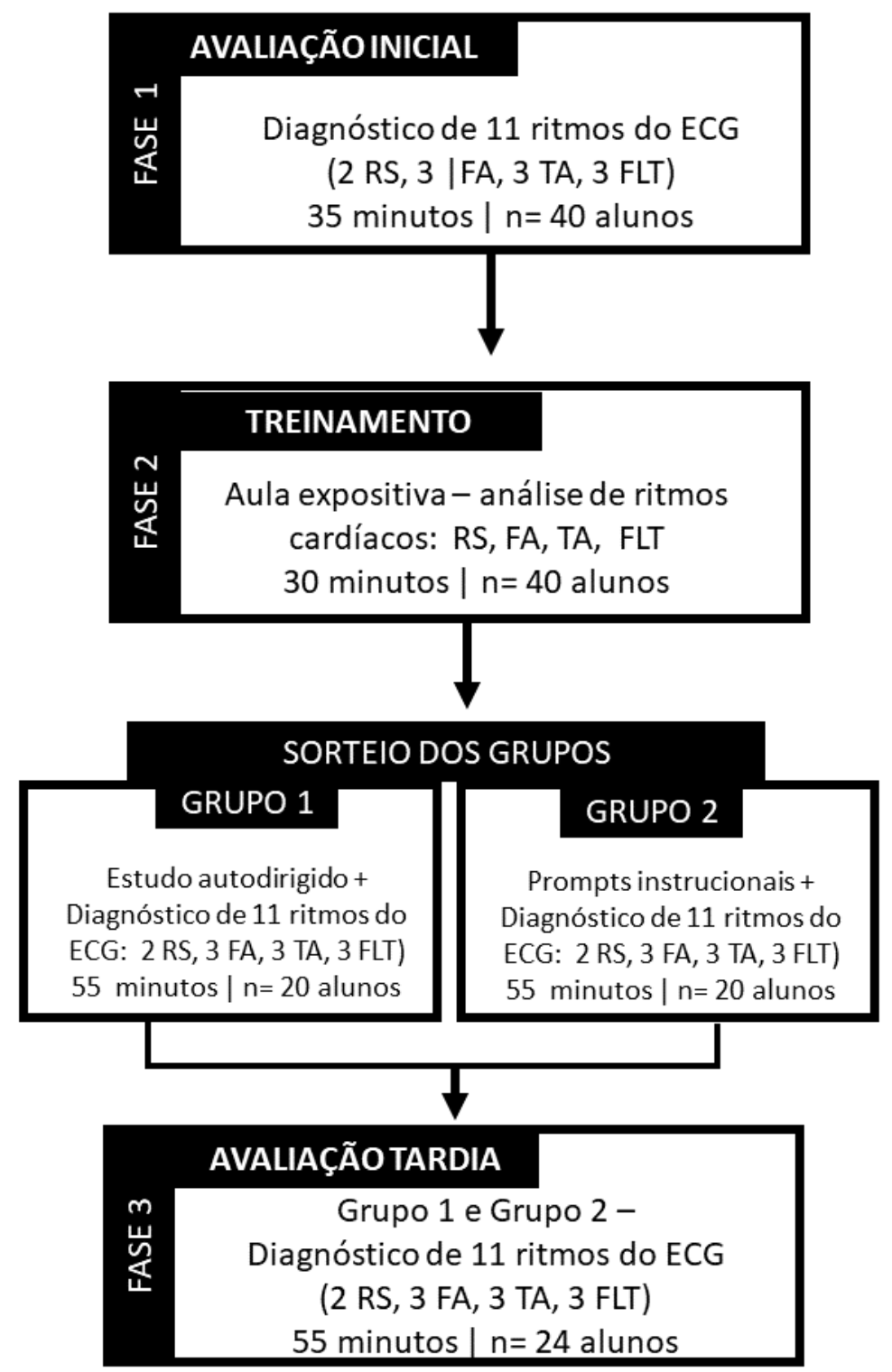

Legenda: $\mathrm{RS}$ = ritmo sinusal; $\mathrm{FA}=$ fibrilação atrial; $\mathrm{TA}=$ taquicardia atrial; FLT = flutter atrial. Fonte: Mello RNB, et al., 2019. 


\section{Local do estudo e participantes}

O estudo foi realizado em uma instituição de ensino superior localizada na cidade de Belo Horizonte que adota o currículo do Aprendizado Baseado em Problemas. Na instituição, o ensino do ECG ocorre no $4^{\circ}$ período, com atividades teórica e prática. No $5^{\circ}$ período os estudantes participam do Bloco de Síndromes Cardiológicas, onde aprendem as alterações patológicas do ECG, incluindo as arritmias que fizeram parte deste estudo.

Foram convidados a participar 40 estudantes do $5^{\circ}$ período antes de iniciarem o Bloco Síndromes Cardiológicas. A escolha desses alunos foi pelo fato de já possuírem conhecimento prévio sobre o ECG normal, mas ainda não tinham sido expostos ao estudo das arritmias que fariam parte do estudo, uma vez que o objetivo era avaliar o ef eito da atividade instrucional planejada sobre a competência diagnóstica para essas arritmias, reduzindo-se assim algum tipo de viés de exposição previa ao tema.

Foram excluídos os alunos que não participaram de todas as fases da pesquisa, aqueles que já haviam cursado o Bloco de Síndromes Cardiológicas e os que não assinaram o Termo de Consentimento Livre e Esclarecido (TCLE).

\section{Materiais}

As arritmias incluídas no estudo foram a Fibrilação Atrial (FA), o Flutter Atrial (FLT) e a Taquicardia Atrial (TA), pelo fato de serem as arritmias supraventriculares mais prevalentes na população, e apesar de apresentarem semelhanças visuais ao ECG, possuem mecanismos eletrofisiológicos distintos. Portanto, contrastá-las é necessário e útil (OLIVEIRA GMM, et al., 2020). O ritmo sinusal (RS) também foi incluído por se tratar do padrão fisiológico de referência.

Para a orientar as atividades, uma semana antes do estudo foi distribuída uma apostila com os conceitos dos ritmos cardíacos incluídos na pesquisa. Utilizou-se como referência a III Diretrizes da Sociedade Brasileira de Cardiologia sobre Análise e Emissão de Laudos Eletrocardiográficos (PASTORE CA, et al., 2016). Foram elaboradas três brochuras com 11 traçados de ECG distintos para cada fase do estudo, contendo cada uma: dois traçados em RS, três em TA, três em FLT e três em FA. Os diagnósticos dos traçados utilizados foram ratificados por três cardiologistas. Um questionário para coleta de dados sociodemográficos foi elaborado para caracterização dos participantes.

\section{Procedimentos}

Na Fase 1 os participantes $(n=40)$ do estudo foram reunidos em uma única sala e após assinarem o TCLE, responderam a um questionário de dados sociodemográficos. A seguir foram convidados a analisar 11 traçados de ECG e informar o diagnóstico do ritmo cardíaco presente em cada um deles (dois traçados em ritmo sinusal, três em fibrilação atrial, três em flutter atrial e três em taquicardia atrial). Os alunos tiveram um tempo de 3 minutos para análise de cada traçado, com uma duração máxima prevista para esta fase de 35 minutos. O tempo gasto para a resolução de cada ECG foi af erido em um estudo piloto realizado previamente.

Na Fase 2, inicialmente os estudantes assistiram a uma aula expositiva, com duração de 30 minutos sobre análise eletrocardiográfica dos ritmos cardíacos que fariam parte do estudo: RS, FA, FLT e TA. A aula foi apresentada por um professor do curso de medicina especialista em arritmias cardíacas e abordou os mecanismos eletrofisiológicos e a interpretação eletrocardiográfica dos ritmos cardíacos citados. A seguir o grupo de estudantes foi dividido por sorteio em dois grupos que foram alocados em salas diferentes.

Os participantes do Grupo $1(n=20)$ e Grupo $2(n=20)$ receberam uma brochura contento onze novos traçados de ECG e deveriam fornecer o diagnóstico do ritmo cardíaco observado. Os alunos tiveram uma duração máxima de 55 minutos para a realização dessa atividade. Era permitido consultar a apostila previamente recebida que continha o conteúdo teórico a respeito dos ritmos cardíacos do estudo. No entanto o Grupo 1, realizou a consulta de forma livre (estudo autodirigido), enquanto os alunos do Grupo 2, receberam um grupo de prompts instrucionais que deveriam ser respondidos antes de informar o diagnóstico eletrocardiográfico final (Quadro 1). 
Quadro 1 - Prompts Instrucionais utilizados no estudo.

\begin{tabular}{|c|c|}
\hline \multicolumn{2}{|r|}{ Analise o traçado do ECG e responda aos comandos abaixo: } \\
\hline Características do ritmo & $\begin{array}{l}\text { 1. Atividade atrial: } \\
\text { - Ondas atriais ausentes: ( ) SIM } \quad \text { ( ) NÃO } \\
\text { - Ondas atriais simétricas: ( ) SIM ( ) NÃO } \\
\text { - Ondas atriais regulares entre si: ( ) SIM ( ) NÃO } \\
\text { - Frequência atrial: ( ) } 100 \text { a } 240 \mathrm{bpm} \text {; ( ) } 250 \text { a } 350 \mathrm{bpm} \text {; ( ) > 350bpm } \\
\text { 2. Ritmo Cardíaco (intervalo R-R): } \\
\text { - Intervalo R-R irreqular: () SIM () NÃO }\end{array}$ \\
\hline & \\
\hline
\end{tabular}

Fonte: Mello RNB, et al., 2019.

Após uma semana, na Fase 3 , os estudantes foram todos reunidos em uma única sala de aula e convidados a fornecer livremente o diagnóstico dos ritmos cardíacos para 11 novos traçados de ECG, com os mesmos ritmos das fases anteriores. O tempo máximo para esta atividade foi de 55 minutos. 0 desfecho primário do estudo foi a acurácia para o diagnóstico do ritmo cardíaco do ECG em cada uma das fases.

\section{Considerações éticas}

O presente estudo foi aprovado pelo Comitê de Ética em Pesquisa, parecer número: 2.702.201 (CAAE: 84230917.4.0000.5143). O TCLE foi apresentado aos estudantes no início da Fase 1, que decidiram livremente pela participação.

\section{Análise dos dados}

Para mensurar a acurácia dos diagnósticos eletrocardiográficos dos participantes, todas os diagnósticos fornecidos pelos estudantes em cada uma das fases do estudo foram listados. Uma vez que as possibilidades diagnósticas se limitavam ao RS, FA, FLT e TA, um sistema de pontuação de 0 a 1 ponto foi adotado, onde 1 ponto foi dado se o diagnóstico estivesse correto e 0 ponto quando o diagnóstico estivesse incorreto. A acurácia diagnóstica final foi obtida pela média dos pontos auferidos em cada uma das fases.

\section{Análise estatística}

Para avaliar a acurácia do diagnóstico eletrocardiográfico dos participantes, foram obtidas três variáveis relacionadas às médias dos escores diagnósticos informados pelos estudantes nas três fases do estudo. Para cada participante, a média da acurácia diagnóstica foi computada em cada uma das fases e para cada um dos grupos (Grupo 1 e Grupo 2). Com o objetivo de avaliar o ef eito das intervenções adotadas em cada grupo e em cada fase do estudo na performance diagnóstica dos alunos para os diagnósticos fornecidos, foi utilizada a Análise de Variância baseada em um planejamento de Medidas Repetidas.

Quando a interação foi significativa no modelo de Análise de Variância foi utilizado o teste de comparações múltiplas de médias Tukey/Duncan/LSD para avaliar as diferenças encontradas dentro de cadaum dos fatores significativos. Os testes de Kolmogorov-Smirnov e de Levene avaliaram respectivamente a normalidade de resíduos e variação constante, verificando os pressupostos da análise. Tendo em vista que os grupos possuíam a mesma distribuição, utilizou-se o teste não paramétrico de Mann-Whitney. O estudo tem 95\% de confiança nas conclusões, sendo a probabilidade de significância inf erior a $5 \%(p<0,05)$.

\section{RESULTADOS}

Quarenta alunos do $5^{\circ}$ período do curso de medicina foram voluntários no estudo, participando das Fases 1 e 2. Para a Fase 3, vinte e sete alunos compareceram, sendo que destes, apenas 24 alunos participaram de todas as fases do estudo. Em relação à caracterização dos participantes, 19 (79,2\%) eram do sexo feminino, 21 (87,5\%) informaram não ter realizado curso de ECG extracurricular, 21 (87,5\%) não faziam parte da Liga Acadêmica de Cardiologia, 12 (50\%) fizeram parte do Grupo 1 e $12(50 \%)$ pertenciam ao Grupo 2.

$\mathrm{Na}$ avaliação inicial realizada na Fase 1, os alunos de ambos os grupos tiveram um índice de acerto muito baixo $(4,16 \%)$. A análise dos resultados evidenciou que houve influência estat isticamente significativa entre 
as fases 1 e 2 do estudo, e, interação significativa entre a Estratégia prompts instrucionais e a Fase 3 na média das notas obtidas pelos alunos (Tabela 1).

Tabela 1 - Influência entre as fases do estudo e o tipo de estratégia utilizada nas notas obtidas pelos alunos ao preencher os diagnósticos do ECG.

\begin{tabular}{lccccc}
\multicolumn{1}{c}{ Fonte de Variação } & $\begin{array}{c}\text { Soma de } \\
\text { quadrados }\end{array}$ & $\mathbf{g . I .}$ & $\begin{array}{c}\text { Quadrados } \\
\text { médios }\end{array}$ & $\mathbf{F}$ & $\boldsymbol{p}$ \\
\hline Estratégia de estudo & 10,125 & 1 & 10,125 & 2,159 & 0,156 \\
\hline Erro (caso) & $\mathbf{1 0 3 , 1 9 4}$ & $\mathbf{2 2}$ & $\mathbf{4 , 6 9 1}$ & - & - \\
\hline Fase do estudo & 165,444 & 2 & 82,722 & 19,974 & $<\mathbf{0 , 0 0 1}$ \\
Fase vs Estratégia de estudo & 30,333 & 2 & 15,167 & 3,662 & $\mathbf{0 , 0 3 4}$ \\
\hline Erro (Fase) & $\mathbf{1 8 2 , 2 2 2}$ & $\mathbf{4 4}$ & $\mathbf{4 , 1 4 1}$ & - & - \\
\hline
\end{tabular}

Nota: Base de dados: 24 alunos; Estratégia de estudo: estudo autodirigido e Prompts Instrucionais; Fases do estudo: Avaliação Inicial, Treinamento e Avaliação Tardia; F = Análise de Variância; $\mathbf{p}=$ Probabilidade de significância do teste; g.I. = grau de liberdade.

Fonte: Mello RNB, et al., 2019.

A Tabela 2, apresenta as médias das notas obtidas para cada grupo de alunos, ao fornecer os diagnósticos dos ritmos do ECG em cada fase do estudo. Observa-se que na Fase 1 (avaliação inicial), as notas obtidas não apresentavam diferenças estatisticamente significativa entre os grupos, o que demonstra que os grupos eram semelhantes no início do estudo.

Tabela 2 - Notas obtidas por grupo ao fornecer os diagnósticos dos ritmos do ECG nas 3 fases do estudo.

\begin{tabular}{cccc}
\hline Fase do estudo & $\begin{array}{c}\text { Estudo autodirigido } \\
\text { Grupo 1 (n=12) } \\
\text { Média } \pm \text { d.p. }\end{array}$ & $\begin{array}{c}\text { Prompts Instrucionais } \\
\text { Grupo 2 (n=12) } \\
\text { Média } \pm \text { d.p. }\end{array}$ & $\boldsymbol{p}$ \\
\hline Fase 1 & $3,3 \pm 2,7$ & $2,2 \pm 1,9$ & $\mathrm{p}=0,427$ \\
Fase 2 & $5,3 \pm 2,3$ & $6,8 \pm 2,2$ & $\mathrm{p}=0,162$ \\
Fase 3 & $4,9 \pm 2,0$ & $6,7 \pm 1,1$ & $\mathbf{p = 0 , 0 2 4}$ \\
\hline $\boldsymbol{p}^{*}$ & $\mathrm{p}^{*}=0,192$ & $\mathbf{p}^{*}<\mathbf{0 , 0 0 1}$ & - \\
\hline
\end{tabular}

Nota: Base de dados: 24 alunos; $p=$ teste de Mann-Whitney; $\boldsymbol{p}^{\star}=$ teste de Friedman; d.p = desvio padrão. Fonte: Mello RNB, et al., 2019.

Em relação à fase do estudo, observa-se que as médias das notas obtidas por ambos os grupos foram maiores na Fase 2 (treinamento) em relação à Fase 1, demonstrando que ambas as estratégias de ensino promoveram aprendizado. Analisando-se a interação a fase de estudo versus estratégia de ensino utilizada, observa-se diferença significativa entre os Grupos, onde na Fase 3 (avaliação tardia), as notas obtidas pelos alunos do Grupo 2, que estudaram os traçados eletrocardiográficos orientados por prompts instrucionais foram superior às dos alunos do Grupo 1 que trabalharam com o estudo autodirigido (Gráfico 1). 


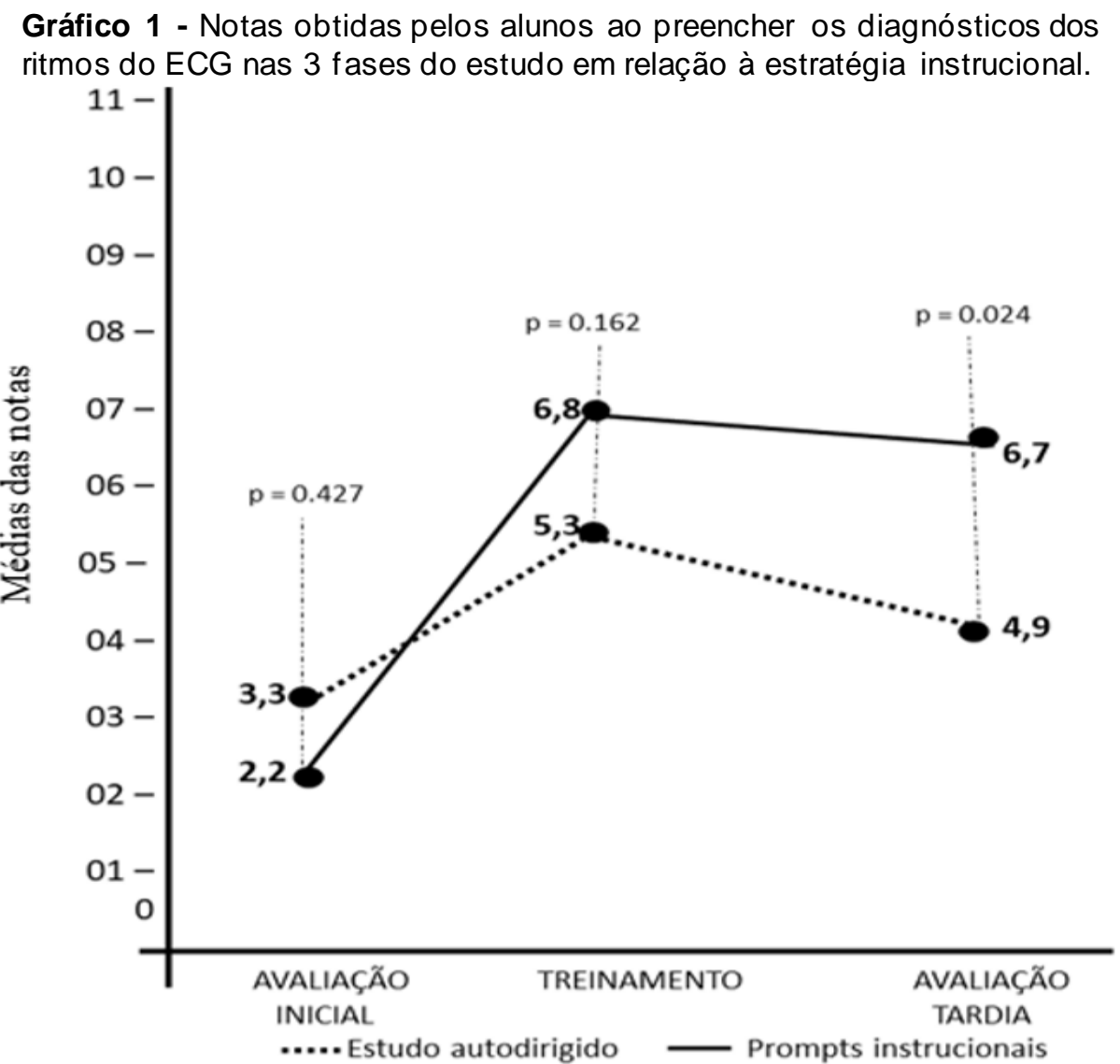

Nota: Base de dados: 24 alunos; $p=$ Teste de Mann-Whitney.

Fonte: Mello RNB, et al., 2019.

\section{DISCUSSÃO}

Este estudo comparou o efeito de duas estratégias de ensino, prompts instrucionais e o estudo autodirigido, na competência diagnóstica de arritmias cardíacas supraventriculares em estudantes de medicina. Os resultados demonstraram que ambas as estratégias melhoraram o desempenho diagnóstico dos estudantes na fase de treinamento, no entanto, o grupo de estudantes que utilizou a estratégia de prompts instrucionais apresentou maior acurácia diagnóstica na fase de avaliação tardia, em relação ao grupo do estudo dirigido.

O ganho de aprendizagem observado na fase de treinamento provavelmente se deve a dois fatores: primeiro o fato de ambos os grupos terem participado de uma aula expositiva sobre as arritmias que fariam parte do estudo, que tinha o objetivo de homogeneizar a amostra de participantes em relação ao nível de conhecimento sobre o tema, e segundo, pela maior exposição aos traçados de ECG promovida por ambas as estratégias de estudo utilizadas.

Apesar de não ter havido um controle do tempo gasto para o estudo de cada ECG na fase de treinamento, a média da acurácia diagnóstica dos ritmos do ECG nesta fase não apresentou diferença significativa entre os grupos, indicando que a aprendizagem adquirida com o treinamento foi homogênea na amostra de participantes. No entanto, a retenção da aprendizagem observada na avaliação tardia foi maior no grupo que trabalhou com os prompts instrucionais, que provavelmente orientou os estudantes em relação às características definidoras e discriminatórias das arritmias, favorecendo que pudessem contrastar os padrões eletrocardiográficos encontrados, contribuindo para o maior aprendizado observado na avaliação tardia.

Os participantes que trabalharam com o estudo autodirigido, apesar da permissão do uso da apostilha, deveriam identificar as características contrastantes dos distúrbios de ritmo por conta própria, sugerindo, que os estudantes deste grupo foram menos eficientes em identificar de modo autônomo os aspectos 
discriminatórios das arritmias cardíacas apresentadas, e que a aula expositiva apenas foi insuficiente para a manutenção da aprendizagem. A dificuldade para a retenção da aprendizagem após uma aula expositiva de ECG foi demonstrada por Raupach T (2016), que identificou uma redução de até $75 \%$ dos escores diagnósticos atingidos pelos estudantes durante uma aula, após oito semanas da atividade.

Ambas as estratégias educacionais comparadas neste estudo desenvolvem a autonomia sobre o processo de aprendizagem. Khiat $\mathrm{H}$ (2015) contudo, notou que a aprendizagem autodirigida apresenta inconsistência tendo em vista a diversidade de experiências estudantis. Nesse sentido, o uso dos prompts instrucionais permite a construção de roteiros de estudo e uma prática orientada, ancorando o desenvolvimento dos novos conhecimentos a partir das habilidades cognitivas já disponíveis. Vygotsky LS (1987) fundamentou uma perspectiva sobre a aprendizagem baseada em uma concepção na qual o conhecimento potencial do estudante, depende de uma conexão ao conhecimento adquirido previamente. Neste aspecto, os prompts instrucionais atuam como andaimes (scaffold) estabelecendo conexões entre o conhecimento que 0 estudante já dispõe e as novas informações.

Estudos em metacognição e aprendizagem autorregulada demonstram que os estudantes muitas vezes não usam espontaneamente as habilidades metacognitivas durante o aprendizado, o que leva a resultados insatisfatórios (SONNENBERG C e BANNERT M, 2015). Os prompts instrucionais utilizam de estruturas que induzem e estimulam as atividades cognitivas, metacognitivas e motivacionais dos alunos durante 0 aprendizado, ativando informações ou habilidades previamente existentes (SONNENBERG C e BANNERT $\mathrm{M}$, 2019). Prompts instrucionais tem por objetivo induzir atividades regulatórias, como orientação, especificação de metas, planejamento, monitoramento, controle, e de avaliação favorecendo o processo de reflexão por parte dos estudantes, que podem assim monitorar seu próprio processo de aprendizagem (MÜLLER NM e SEUFERT T, 2018)

Provavelmente, as habilidades cognitivas necessárias para o aprendizado dos distúrbios do ritmo do ECG, nos alunos deste estudo, ainda se encontravam em um nível de desenvolvimento que requeria alguma forma de suporte instrucional, para que a aprendizagem pudesse se desenvolver. O prompts instrucionais atuaram como "andaimes" no processo da aprendizagem dos estudantes que utilizaram desta estratégia, favorecendo o desenvolvimento de suas habilidades potenciais.

É importante salientar que esse trabalho apresenta limitações por se tratar, até onde se sabe, de estudo único com uso de prompts instrucionais para o ensino do ECG, com pequeno número de participantes e aplicado a apenas três tipos de distúrbio do ritmo cardíaco. Assim, a sua replicação para outros tipos de análise eletrocardiográfica e com maior número de estudantes poderá contribuir para a consolidação dos achados. No entanto o estudo oferece contribuições conceituais importantes para a desafiadora missão do ensino do ECG, apresentando uma ferramenta cognitiva simples e de baixo custo que pode ser implementada nos diversos cenários da educação em saúde.

Cabe ressaltar que o método pode ser aplicado para o ensino na interpretação eletrocardiográfica de diferentes distúrbios do ritmo cardíaco, preferencialmente aqueles que apresentam características que devam ser contrastadas. Neste estudo foram utilizadas arritmias visualmente semelhantes entre si, mas com distintos mecanismos eletrofisiológicos, cuja análise da atividade atrial auxilia no diagnóstico diferencial. Por exemplo, é possível utilizar os prompts instrucionais para a análise das taquicardias de QRS largo, diferenciando a taquicardia ventricular da taquicardia supraventricular com aberrância de condução. Neste caso já existem os algoritmos de Brugada e Vereckei, que são instruções normatizadas para a diferenciação destes distúrbios de ritmos, no entanto, estes algoritmos podem ser apresentados através de prompts instrucionais durante uma atividade de ensino (PASTORE CA, et al.,2016).

O método pode ser incorporado em diversos contextos educacionais onde é necessária a diferenciação eletrocardiográfica de ritmos semelhantes entre si, como entre taquicardia reentrante AV ortodrômica e taquicardia por reentrada nodal, bem como na diferenciação entre arritmia sinusal e bloqueio sinoatrial de segundo grau, ou em eventos contrastantes como os bloqueios divisionais do ramo esquerdo versus ramo direito. Entre os bloqueios atrioventriculares de $1^{\circ}, 2^{\circ}$ e $3^{\circ}$ graus. Assim como na diferenciação das sobrecargas de câmara cardíacas. 


\section{CONCLUSÃO}

O uso de prompts instrucionais para o ensino das arritmias cardíacas que fizeram parte deste estudo, melhorou a acurácia diagnóstica dos estudantes em comparação à estratégia do estudo autodirigido. Os prompts instrucionais favoreceram uma aprendizagem significativa para os diagnósticos dos ritmos cardíacos estudados pelos participantes. A estratégia proposta é de fácil execução e pode ser considerada para o ensino e desenvolvimento do raciocínio eletrocardiográfico em cursos de graduação e pós-graduação.

\section{REFERÊNCIAS}

1. ABREU GCF, et al. Efeito da elaboração de mapas conceituais na acurácia diagnóstica de estudantes de medicina. Revista Eletrônica Acervo Saúde, 2021;13(2):e6150.

2. ANTIPEROVITCH P, et al. Proposed In-Training Electrocardiogram Interpretation Competencies for Undergraduate and Postgraduate Trainees. Journal of Hospital Medicine, 2018;1;13(3):185-193.

3. BARROS MNDS, et al. New ECG training methodology: demystifying theory in practice: Practical teaching of ECG. Revista Brasileira de Educação Médica, 2015;40: 751-756.

4. BOJSEN SR, et al. The acquisition and retention of ECG interpretation skills after a standardized web-based ECG tutorial- a randomized study. BMC Medical Education, 2015, 15: 1- 9.

5. BARAL R, et al. The effectiveness of a nationwide interactive ECG teaching workshop for UK medical students. Journal of Electrocardiology, 2020;58:74-79.

6. BREEN CJ, et al. ECG interpretation skill acquisition: A review of learning, teaching and assessment. Journal of Electrocardiology, 2019;S0022-0736(18):30641-1

7. COOK DA, et al. Accuracy of physicians' electrocardiogram interpretations: a systematic review and meta -analysis. JAMA Internal Medicine, 2020;180(11):1461-1471.

8. CHAMBERLAND M, MAMEDE, S. Self-Explanation: an instructional strategy to foster clinical reasoning in medical students. Health Professions Education, 2015;1(1): 24-33.

9. FENT G, et al. Teaching the interpretation of electrocardiograms: which method is best? Journal of Electrocardiology, 2015;48: 190-193

10. KEWCHAROEN J, et al. A comparison between peer-assisted learning and self-study for electrocardiography interpretation in Thai medical students. Journal in Advances of Medical Education and Professionalism, 2020;8(1):1824.

11. KHIAT H. Measuring Self-Directed Learning: a diagnostic tool for adult learners. Journal of University Teaching \& Learning Practice, 2015;12(2): 1-159.

12. KOPEC G, et al. Competency in ECG Interpretation Among Medical Students. Medical Science Monitor, 2015; 21: 3386-3394.

13. KOPEC G, et al. Electrocardiogram reading: a randomized study comparing 2 e-learning methods for medical students. Polish Archives of Internal Medicine, 2018;128(2): 98-104

14. KRASNE S, et al. Mastering Electrocardiogram Interpretation Skills Through a Perceptual and Adaptive Learning Module. AEM Education and Training, 2020;5(2):e10454.

15. MÜLLER NM, SEUFERT T. Effects of self-regulation prompts in hypermedia learning on learning performance and self-efficacy. Learning and Instruction, 2018;58:1-11

16. NASCIMENTO BR, et al. cardiovascular disease epidemiology in Portugu ese-speaking countries: data from the Global Burden of Disease, 1990 to 2016. Arquivos Brasileiros de Cardiologia, 2018;110(6):500-511.

17. OLIVEIRA GMM, et al. Estatística Cardiovascular - Brasil, 2020. Arquivos brasileiros de Cardiologia, 2020; 115(3): 308-439.

18. PAUL B, BARANCHUK A. Electrocardiography Teaching in Canadian Family Medicine Residency Programs: A National Survey. Family Medicine, 2011;43(4): 267-271.

19. PASTORE CA, et al. III Diretrizes da Sociedade Brasileira de Cardiologia sobre Análise e Emissão de Laudos Eletrocardiográficos. Arquivos Brasileiros de Cardiologia, 2016;106(4) - suplemento: 1-23.

20. ROJAS FL, et al. Electrocardiographic abnormalities in Chagas disease in the general population: a systematic review and meta-analysis. PLoS Neglected Tropical Diseases, 2018;12(6): e0006567.

21. RAUPACH T, et al. How can we improve teaching of ECG interpretation skills? Findings from a prospective randomised trial. Journal of Electrocardiology, 2016, 49:7-12.

22. SURESH K, et al. Evaluation on ECG training program for interns and postgraduates. Journal of Evolution of medical and Dental Sciences, 2018;7(4): 407-410.

23. SONNENBERG C, BANNERT M. Discovering the effects of metacognitive prompts on the sequential structure of SRLprocesses using process-mining techniques. Journal of Learning Analytics, 2015, 2(1):72-100.

24. SONNENBERG C, BANNERT M. Using Process Mining to examine the sustainability of instructional support: How stable are the effects of metacognitive prompting on self-regulatory behavior? Computer in Human Behavior, 2019; 96: 259-272.

25. VILJOEN CA, et al. Is computer-assisted instruction more effective than other educational methods in achieving ECG competence amongst medical students and residents? A systematic review and meta-analysis. BMJ Open, 2019; 9(11): e028800.

26. VILJOEN CA, et al. Effectiveness of blended learning versus lectures alone on ECG analysis and interpretation by medical students. BMC Medical Education, 2020;20(1): 488.

27. VYGOTSKY LS. Pensamento e linguagem. São Paulo: Martins Fontes, 1987;212p.

28. $\mathrm{XU} \mathrm{H}$, et al. Methods to Improve Diagnostic Reasoning in Undergraduate Medical Education in the Clinical Setting:a Systematic Review. Journal of General Internal Medicine, 2021;36(9):2745-2754. 